\title{
$\beta$-Lactam Antibiotic Production by Streptomyces clavuligerus Mutants Impaired in Regulation of Aspartokinase
}

\author{
By SIMONA MENDELOVITZ AND YAIR AHARONOWITZ* \\ Tel Aviv University, George S. Wise Faculty of Life Sciences, Department of Microbiology, \\ Tel Aviv, Israel 69978
}

(Received 17 August 1982; revised 10 December 1982)

\begin{abstract}
This work describes isolation and characterization of Streptomyces clavuligerus mutants resistant to the lysine analogue $S$-(2-aminoethyl)-L-cysteine (AEC). The mutation to AEC resistance was shown to affect the feedback regulation of aspartokinase; $70 \%$ of the mutants isolated had aspartokinase activity insensitive to concerted feedback inhibition by lysine plus threonine. Among these mutants, $70 \%$ (about $50 \%$ of the total AEC-resistant strains isolated) showed significant overproduction of $\beta$-lactam antibiotics.
\end{abstract}

\section{INTRODUCTION}

The cephalosporins, cephamycin, and the penicillin $\mathrm{N}$ types of $\beta$-lactam antibiotics, which are produced by fungi and streptomycetes are derived mainly from three amino acids: $\alpha$ aminoadipic acid (AAA), cysteine and valine. Extensive physiological, biochemical and genetic studies have revealed the direct involvement of primary metabolites in the regulation of $\beta$ lactam antibiotic production (Drew \& Demain, 1977). The metabolic origin of the AAA moiety of $\beta$-lactam antibiotic produced by fungi differs from that of the streptomycetes; in fungi AAA is an intermediate of the lysine biosynthetic pathway (Umbarger, 1978), whereas in streptomycetes AAA originates as a catabolic product of lysine produced from the diaminopimelic acid (DAP)-lysine branch of the aspartate pathway (Kirkpatrick et al., 1973; Kern et al., 1980). Therefore, the involvement of lysine metabolism in controlling $\beta$-lactam antibiotic synthesis in fungi and streptomycetes becomes of special interest, from both a comparative biochemical as well as the practical point of view.

Suppression of penicillin formation by addition of L-lysine to cultures of Penicillium chrysogenum was first observed by Demain (1957). It has been attributed to feedback regulatory effects of lysine on homocitrate synthetase, the first step in the fungal lysine biosynthetic pathway (Masurekar \& Demain, 1972; Luengo et al., 1980). In contrast, addition of L-lysine and a mixture of DL- and meso-DAP to a culture of Streptomyces clavuligerus in chemically defined medium significantly stimulated $\beta$-lactam antibiotic production (Mendelovitz \& Aharonowitz, 1982). It therefore became interesting to see whether alteration of the intracellular lysine pool by genetic means could affect $\beta$-lactam antibiotic production in a similar fashion to that obtained with external lysine addition. The use of lysine antimetabolites for selection of lysine-regulatory mutants was chosen to achieve this goal. Masurekar \& Demain (1974) were able to obtain lysineregulatory mutants of $P$. chrysogenum which overproduced and excreted lysine and were deficient in production of penicillin. Further, a revertant of such a mutant was found to recover the ability to produce penicillin. Bacterial mutants resistant to lysine analogues have already been used commercially for lysine production. Among these, mutants of Brevibacterium flavum resistant to $S$-(2-aminoethyl)-L-cysteine (AEC) produced lysine most effectively (Sano \& Shiio, 1970).

Abbreviations: AAA, $\alpha$-aminoadipic acid; AEC, $S$-(2-aminoethyl)-L-cysteine; AK, aspartokinase; DAP, diaminopimelic acid; DDPS, dihydrodipicolinate synthetase. 
The objective of this work was to select for AEC-resistant mutants of $S$. clavuligerus, to investigate possible alteration in regulation of their lysine biosynthetic pathway, and to determine whether such a mutation might affect cephamycin formation. As will be shown, $70 \%$ of AEC-resistant mutants of $S$. clavuligerus, in which feedback regulation of aspartokinase activity was impaired, produced elevated levels of antibiotics.

\section{METHODS}

Bacterial cultivation. Streptomyces clavuligerus NRRL 3585 (Higgens \& Kastner, 1971) was used throughout this study. The chemically defined medium and growth conditions employed were reported previously (Aharonowitz \& Demain, 1977). Selection of analogue-resistant mutants was carried out on agar containing all minimal medium components except asparagine. The latter was replaced by $40 \mathrm{~mm}-\mathrm{NH}_{4} \mathrm{Cl}$ as nitrogen source. Spores were prepared on solid medium which contained $\left(1^{-1}\right): 10 \mathrm{~g}$ malt extract (Difco), $4 \mathrm{~g}$ yeast extract, $4 \mathrm{~g}$ glycerol, $100 \mu \mathrm{g}$ $\mathrm{CoCl}_{2}$, and $20 \mathrm{~g}$ agar, adjusted to $\mathrm{pH} 7 \cdot 0$.

Isolation of AEC-resistant mutants. Spores suspended in $1 \mathrm{ml} 50 \mathrm{~mm}$-Tris/HCl buffer, $\mathrm{pH} 7 \cdot 5$, containing $0.01 \%$ (v/v) Triton X-100 were transferred to an Erlenmeyer flask $(125 \mathrm{ml})$ containing $7.5 \mathrm{ml} 5.0 \mathrm{~mm}-\mathrm{Tris} / \mathrm{HCl}$ buffer, pH 7.5. $N$-Methyl- $N^{\prime}$-nitro- $N$-nitrosoguanidine was added to a final concentration of $0.5 \mathrm{mg} \mathrm{ml}^{-1}$. The mixture was agitated momentarily and incubated in a gyrotory shaker at $30^{\circ} \mathrm{C}$. Samples were taken, at time intervals, washed by centrifugation and spread on agar plates containing defined medium (see above) and AEC at a concentration of $1 \mathrm{mg} \mathrm{ml}^{-1}$. Colonies which developed within one week were isolated, restreaked on sporulation medium and tested for resistance to AEC.

Aspartokinase assay. Aspartokinase activity was measured by the formation of aspartylhydroxamate (Stadtman et al., 1961) with the $30-65 \%$ saturated $\left(\mathrm{NH}_{4}\right)_{2} \mathrm{SO}_{4}$ fraction of crude sonic extracts. The reaction mixture contained (in $1 \mathrm{ml}$ ): $0.1 \mathrm{M}$-Tris/ $\mathrm{HCl}$ buffer $\mathrm{pH} 7.6,0.02 \mathrm{M}$-ATP, $0.02 \mathrm{M}$-aspartate, $0.01 \mathrm{M}-\mathrm{MgSO}_{4}, 1.4 \mathrm{~mm}-\beta$ mercaptoethanol, $0 \cdot 8 \mathrm{M}-\mathrm{NH}_{2} \mathrm{OH} . \mathrm{HCl}$ neutralized with $\mathrm{KOH}$, and enzyme as indicated. Incubation was at $30^{\circ} \mathrm{C}$. Specific activity was expressed as nmol aspartylhydroxamate formed $\mathrm{min}^{-1}$ ( $\mathrm{mg}$ protein $)^{-1}$.

Fermentations. All cultures were grown in $250 \mathrm{ml}$ Erlenmeyer flasks containing $50 \mathrm{ml}$ of the chemically defined medium described by Aharonowitz \& Demain (1977). Incubation was at $30^{\circ} \mathrm{C}$ for $120 \mathrm{~h}$ on a gyrotory shaker with a 2 in $(5 \mathrm{~cm})$ stroke at 250 r.p.m.

Antibiotic assay. Samples were assayed for the production of cephalosporins by the standard disc agar plate technique, using as an assay organism an Escherichia coli strain Ess that is supersensitive to $\beta$-lactam antibiotics (Kohsaka \& Demain, 1976). In this assay we detected only the cephamycin C, which was the dominant product (as was confirmed by HPLC analysis). Clavulanic acid had only $5-10 \%$ of the activity of cephalosporin $\mathrm{C}$ in the assay (Aharonowitz \& Demain, 1977).

\section{RESULTS}

\section{Effect of AEC on growth and aspartokinase activity}

When the wild-type strain was grown on a solid chemically defined medium containing increasing concentrations $\left(>1 \mathrm{mg} \mathrm{ml}^{-1}\right)$ of the lysine analogue AEC, growth was completely inhibited. However, in liquid medium of the same composition, the concentration required to achieve $50 \%$ inhibition of growth was $1.5 \mathrm{mg} \mathrm{ml}^{-1}$. Addition of L-lysine, at concentrations four times lower than that of the inhibitor, reversed the inhibitory effect of AEC and allowed normal growth to occur.

Since aspartokinase (AK) activity of $S$. clavuligerus is under concerted feedback inhibition by lysine plus threonine (Mendelovitz \& Aharonowitz, 1982), the possible substitution of lysine by its analogue AEC and hydroxylysine in the AK assay was investigated. The results in Table 1 demonstrate feedback inhibition of AK activity by the concerted effect of threonine plus AEC, similar to that achieved by threonine plus lysine. The combination of threonine plus hydroxylysine inhibited AK activity to a lesser extent. It was assumed that addition of threonine to the AEC-containing growth medium would increase the growth inhibitory effect mediated by AEC. However, threonine actually increased the inhibitory effect of AEC only over a very narrow range of concentrations $\left(20-200 \mu \mathrm{g} \mathrm{ml}^{-1}\right.$; Fig. 1); upon further increase of threonine concentration, the inhibitory effect of AEC was reversed.

\section{Regulation of $A K$ activity in the AEC-resistant mutants}

Each of the AEC-resistant colonies was tested for growth, AK activity and antibiotic production. Although most colonies tested appeared morphologically similar, differences in 
Table 1. Effect of threonine, lysine and lysine analogues on aspartokinase activity

The standard hydroxamate assay was used. The enzyme preparation was the 30 to $65 \%$ ammonium sulphate fraction prepared from a $48 \mathrm{~h}$ culture. Protein content in each assay mixture was $0.94 \mathrm{mg}$. In a control reaction, with no added amino acid, $21.6 \mathrm{nmol}$ aspartohydroxamate $\min ^{-1}$ were formed, representing $100 \%$ relative activity.

\begin{tabular}{lc}
\multicolumn{1}{c}{$\begin{array}{c}\text { Addition } \\
(5 \mathrm{mM})\end{array}$} & $\begin{array}{c}\text { Relative enzyme } \\
\text { activity } \\
(\%)\end{array}$ \\
None & 100 \\
L-Threonine & 106 \\
L-Lysine & 140 \\
Hydroxylysine & 95 \\
$S$-(2-aminoethyl)-L-Cysteine (AEC) & 118 \\
L-Lysine + L-threonine & 6 \\
AEC + L-threonine & 18 \\
Hydroxylysine + L-threonine & 64
\end{tabular}

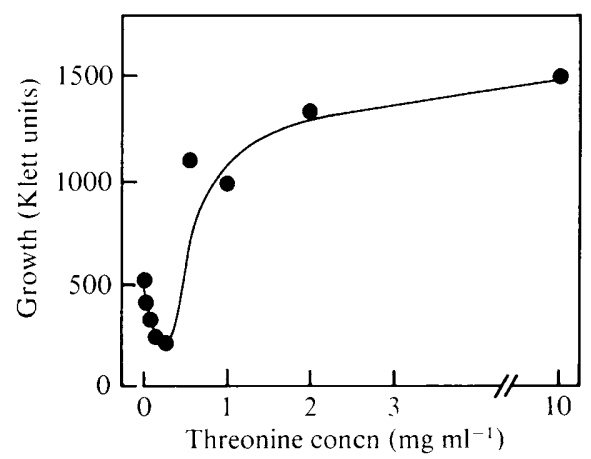

Fig. 1. Effect of threonine on growth of $S$. clavuligerus in presence of AEC. Cells were grown in the chemically defined medium containing $1 \mathrm{mg} \mathrm{AEC} \mathrm{ml}^{-1}$ and increasing concentrations of threonine. Maximum growth at $66 \mathrm{~h}$ observed in the control culture was $1250 \mathrm{Klett}$ units.

their growth patterns were observed in the liquid medium. Some of the mutants were poor growers and some formed dense clumps. Of the several different genotypes that could account for the AEC resistance phenotype, those which possessed a genetic alteration in the AK regulatory site were of special interest. It was expected that in such mutants the concerted inhibitory effect exerted by threonine plus lysine (or its analogues) on AK activity would be absent. Accordingly, a cell extract from each AEC-resistant mutant obtained was tested for AK activity in the presence and absence of the putative feedback inhibitors. In 32 out of the 44 mutants tested, AK activity differed from that of the wild-type in that it was unaffected by concentrations of lysine plus threonine that caused about $50 \%$ inhibition of AK activity in the wild-type strain. Table 2 presents data on 12 such mutants resistant to AEC. The AK activity of another three mutants (strains 1, 20 and 38) was inhibited in the presence of these two amino acids (Table 2). AK activity, in presence of $8 \mathrm{~mm}$-lysine, was always higher than in the control (no addition), the degree of stimulation being between $20-100 \%$. Significant differences in AK activity were usually also obtained in the presence of $8 \mathrm{~mm}$-threonine. These changes in $\mathrm{AK}$ activity provoked by threonine might be explained by differences in enzyme concentration in different reaction mixtures (Shabtai et al., 1975).

Two mutants (strains 11 and 52) were assayed for repression and inhibition of dihydrodipicolinate synthetase (DDPS) by lysine, DAP, isoleucine, homoserine, methionine, AAA and lysine. No change with respect to feedback regulation of DDPS in the mutant compared to the wild-type was observed; DAP repressed DDPS synthesis by $30 \%$, whereas DAP and AAA each inhibited DDPS activity by $30 \%$. 
Table 2. Effect of $\mathrm{L}-$ lysine and $\mathrm{L}-$ threonine on aspartokinase activity in AEC-resistant mutants

Organisms were grown in chemically defined medium and harvested in their late growth phase (48$72 \mathrm{~h}$ ). Aspartokinase activity was assayed in the ammonium sulphate fraction as described in the legend to Table 1.

\begin{tabular}{|c|c|c|c|c|c|}
\hline \multirow[b]{2}{*}{ Mutant no. } & \multicolumn{4}{|c|}{ Relative aspartokinase activity in the presence of: } & \multirow[b]{2}{*}{$\begin{array}{l}\text { Aspartokinase } \\
\text { deregulation }\end{array}$} \\
\hline & $\begin{array}{c}\text { No } \\
\text { addition }\end{array}$ & $\begin{array}{c}\text { L-Lysine } \\
(8 \mathrm{mM})\end{array}$ & $\begin{array}{l}\text { L-Threonine } \\
(8 \mathrm{~mm})\end{array}$ & $\begin{array}{c}\text { L-Lys }(0.4 \mu \mathrm{M}) \\
+ \text { L-Thr }(0.4 \mathrm{mM})\end{array}$ & \\
\hline Wild-type & 100 & 173 & 115 & 35 & - \\
\hline $\operatorname{aec}(\mathbf{R}) 1$ & 100 & 141 & 71 & 35 & - \\
\hline $\operatorname{aec}(\mathbf{R}) 3$ & 100 & 142 & 93 & 100 & + \\
\hline$a e c(\mathbf{R}) 5$ & 100 & 127 & 110 & 100 & + \\
\hline $\operatorname{aec}(\mathrm{R}) 10$ & 100 & 129 & 109 & 98 & + \\
\hline $\operatorname{aec}(\mathbf{R}) 11$ & 100 & 139 & 104 & 100 & + \\
\hline$a e c(\mathrm{R}) 15$ & 100 & 124 & 109 & 90 & + \\
\hline $\operatorname{aec}(\mathrm{R}) 20$ & 100 & 151 & 87 & 29 & - \\
\hline $\operatorname{aec}(\mathrm{R}) 22$ & 100 & 159 & 100 & 93 & + \\
\hline $\operatorname{aec}(\mathrm{R}) 27$ & 100 & 178 & 119 & 95 & + \\
\hline$a e c(\mathrm{R}) 28$ & 100 & 148 & 203 & 98 & + \\
\hline $\operatorname{aec}(\mathrm{R}) 35$ & 100 & 219 & 122 & 142 & + \\
\hline $\operatorname{aec}(\mathrm{R}) 38$ & 100 & 161 & 104 & 61 & - \\
\hline $\operatorname{aec}(\mathrm{R}) 50$ & 100 & 193 & 192 & 116 & + \\
\hline $\operatorname{aec}(\mathrm{R}) 52$ & 100 & 128 & 117 & 107 & + \\
\hline $\operatorname{aec}(\mathrm{R}) 54$ & 100 & 146 & 143 & 114 & + \\
\hline
\end{tabular}

Table 3. Specific antibiotic production by AEC-resistant and wild-type strains of $S$. clavuligerus

Cultures were grown in chemically defined medium without any added amino acids except asparagine, which served as the nitrogen source. At various times, samples were taken for $\mathrm{pH}$, biomass, and antibiotic analysis.

\begin{tabular}{|c|c|c|c|c|c|}
\hline Mutant no. & $\begin{array}{l}\text { Aspartokinase } \\
\text { deregulation* }\end{array}$ & $\begin{array}{c}\text { Dry cell } \\
\text { wt } \\
\left(\mathrm{mg} \mathrm{ml}^{-1}\right)\end{array}$ & $\begin{array}{l}\text { Maximum } \\
\text { antibiotic } \\
\text { concn } \\
\left(\mu \mathrm{g} \mathrm{ml}^{-1}\right)\end{array}$ & $\begin{array}{c}\text { Specific production } \\
\text { of antibiotic } \ddagger \\
{\left[\mu \mathrm{g}(\mathrm{mg} \text { dry cell } w \mathrm{t})^{-1}\right]}\end{array}$ & $\begin{array}{l}\text { Relative } \\
\text { specific } \\
\text { production } \\
\text { value }\end{array}$ \\
\hline Wild-type & - & 1.8 & 84 & 47 & $1 \cdot 0$ \\
\hline $\operatorname{aec}(\mathbf{R}) 3$ & + & 0.8 & 95 & 122 & $2 \cdot 6$ \\
\hline $\operatorname{aec}(\mathbf{R}) 5$ & + & $1 \cdot 2$ & 260 & 217 & $4 \cdot 6$ \\
\hline $\operatorname{aec}(\mathbf{R}) 10$ & + & $1 \cdot 3$ & 122 & 94 & $2 \cdot 0$ \\
\hline $\operatorname{aec}(\mathbf{R}) 11$ & + & 0.7 & 127 & 181 & $3 \cdot 9$ \\
\hline $\operatorname{aec}(\mathbf{R}) 15$ & + & $1 \cdot 3$ & 420 & 323 & 6.9 \\
\hline $\operatorname{aec}(\mathbf{R}) 22$ & + & $1 \cdot 3$ & 285 & 219 & $4 \cdot 9$ \\
\hline $\operatorname{aec}(\mathbf{R}) 27$ & + & 1.5 & 210 & 140 & $3 \cdot 0$ \\
\hline $\operatorname{aec}(\mathbf{R}) 28$ & + & $1 \cdot 1$ & 345 & 313 & $6 \cdot 7$ \\
\hline $\operatorname{aec}(\mathbf{R}) 35$ & + & 1.4 & 150 & 107 & $2 \cdot 3$ \\
\hline $\operatorname{aec}(\mathrm{R}) 38$ & - & 1.8 & 49 & 27 & 0.6 \\
\hline $\operatorname{aec}(\mathbf{R}) 50$ & + & 1.5 & 405 & 270 & 5.7 \\
\hline $\operatorname{aec}(\mathbf{R}) 52$ & + & $1 \cdot 2$ & 285 & 237 & $5 \cdot 0$ \\
\hline $\operatorname{aec}(\mathbf{R}) 54$ & + & $1 \cdot 1$ & 230 & 209 & $4 \cdot 4$ \\
\hline
\end{tabular}

\section{Antibiotic production by the AEC-resistant mutants}

All AEC-resistant mutants were grown in the chemically defined medium (four independent growth cycles) for $120 \mathrm{~h}$ and broth samples were assayed for antibiotic. Among those mutants in which AK activity was deregulated, 23 out of 32 (about $70 \%$ ) produced antibiotic at higher titres and at higher specific production values $\left.[\mu \mathrm{g} \text { antibiotic ( } \mathrm{mg} \text { dry cell weight })^{-1}\right]$ than the wild-type. 
In fermentations of the other nine cultures, production values did not significantly differ from those of the wild-type. Among the AEC-resistant mutants possessing a non-deregulated AK activity, 3 out of 12 tested $(25 \%)$ still yielded improved titres (between 120 and $280 \mu \mathrm{g} \mathrm{ml}^{-1}$ ) corresponding to $2-5$ times more antibiotic produced based on specific production values. The results shown in Table 3 represent fermentation data for 12 AEC-resistant mutants deregulated in their AK activity and one which was not deregulated. The antibiotic titres achieved in these fermentations ranged from $1 \cdot 1$ to 5.9 times higher than the wild-type titre. Furthermore, due to the decrease in the maximum biomass obtained with the AEC-resistant mutants, the calculated relative specific production values of those strains were even higher. Mutant aec $(R) 38$, the one which retained a normal AK regulation pattern, could grow to the same extent as the wild-type. However, its capacity to produce antibiotics was significantly lower.

\section{DISCUSSION}

Our results show that superior $\beta$-lactam antibiotic-producing strains of $S$. clavuligerus were prevalent among mutants selected for resistance to the lysine analogue, AEC. Although several genotypes can explain the AEC-resistant phenotype, genetic alteration which results in an impaired regulation of $\mathrm{AK}$ activity was of particular interest to us.

The AK activity of $S$. clavuligerus was previously shown to be feedback-inhibited by the concerted effect of threonine plus lysine (Mendelovitz \& Aharonowitz, 1982). Lysine could be replaced by AEC for concerted feedback inhibition of AK activity (Table 1). Since AEC also inhibited growth of $S$. clavuligerus, an effect that could be reversed by lysine addition, it seemed reasonable to assume that AEC acted as a lysine antimetabolite. Presumably then, the combination of AEC and threonine in the growth medium should have enhanced growth inhibition. However, such an effect was only observed at low threonine concentrations; when threonine concentration was increased, restoration of normal growth levels was observed. The reason for this phenomenon is unclear. However, observations reported by Gross \& Burkhardt (1973) suggest possible involvement of amino acid transport mechanisms. Two uptake systems for lysine, arginine and their analogues exist in Streptomyces hydrogenans (Gross \& Burkhardt, 1973). One system operates when low $(<0.1 \mathrm{~mm})$ extracellular lysine (or lysine analogue) concentrations are available, whereas when higher lysine and arginine concentrations are available, lysine is taken up by a second system which generally mediates the uptake of neutral amino acids. This latter system has a lower affinity for lysine and arginine, but its transport capacity is higher than the first, lysine-specific, system. Since we have used $5 \mathrm{mM}$-AEC in order to inhibit $S$. clavuligerus growth, the second transport system was probably operating. Thus, upon increasing threonine concentration, AEC uptake could be competitively inhibited.

It was the in vivo effect of AEC that enabled selection of AEC-resistant mutants of the sort from which AK-deregulated strains could be screened. Indeed, such mutants occurred at high frequency among the AEC-resistant types (Table 2).

The data presented here suggest a similarity between the AK systems of $S$. clavuligerus and Brevibacterium flavum. In B. flavum, AK activity appears to be due to a single enzyme which is concertedly feedback-inhibited by lysine plus threonine (Shiio \& Miyajima, 1969); AECresistant mutants of the $B$. flavum strain were found to possess a desensitized AK free from feedback inhibition by lysine plus threonine, and also produced lysine in excess (Sano \& Shiio, 1970).

Since L-lysine and DL-meso-DAP each stimulated specific $\beta$-lactam antibiotic production by $S$. clavuligerus (Mendelovitz \& Aharonowitz, 1982), and since feedback control was exerted by lysine plus threonine on AK, it was assumed that carbon flow from aspartate to lysine and AAA would be rate-limiting for antibiotic synthesis. Therefore, increased antibiotic synthesis was expected upon release of enzymes involved in lysine biosynthesis from such feedback effects. Consistent with this idea was the finding that $70 \%$ of the mutants impaired in the feedback regulation of their AK activity (about $50 \%$ of the total AEC-resistant population) produced significantly higher amounts of antibiotics, in terms of both volumetric titre and specific production. However, with respect to antibiotic production capacity, other phenotypes were 
also found among the AEC-resistant strains, specifically: $(a)$ those which were deregulated in their AK activity but did not produce higher amounts of antibiotics $(20 \%$ of the total $) ;(b)$ those which were unaffected in AK activity, but did produce high amounts of antibiotics $(6 \%$ of the total); and (c) those which possessed an unaffected AK activity and produced normal amounts of antibiotics $(20 \%$ of the total). Types $(b)$ and $(c)$ could be the result of genetic alterations in genes responsible for AEC transport or AEC modification. The reason why type $(b)$ mutants are antibiotic producers is, however, still unclear.

Although the majority of the AEC-resistant AK-deregulated mutants produce higher antibiotic titres as well as higher specific production values, the degree of increase did not correlate exactly with the degree of impairment in AK regulation. The possible involvement of more than a single mutation could not be ruled out. Further analysis of biochemical defects in the AK protein extracted from different mutants is still required for a better understanding of its role in regulating the carbon flow towards the lysine branch of the aspartate pathway. Two other enzymic activities in the lysine branch might be suitable targets for regulation. One, DAPdecarboxylase, was suggested to be under regulation in Streptomyces lipmanii (Kirkpatrick et al., 1973 ) and is now under study in S. clavuligerus in our laboratory. As was suggested by Kern et al. (1980), a second possible target for regulation in the pathway leading to $\alpha$-aminoadipate is Llysine $\varepsilon$-aminotransferase.

The rationale behind the isolation of lysine analogue-resistant mutants was to obtain lysine overproducers. Such mutants of $P$. chrysogenum overproduced lysine and underproduced penicillin (Masurekar \& Demain, 1974). In sharp contrast, corresponding mutants of $S$. clavuligerus were found to overproduce cephalosporins.

The link between primary and secondary metabolism shown in this work demonstrates the feasibility of obtaining strains that produce large amounts of antibiotics through selection for mutants deregulated in the biosynthesis of a primary metabolite directly involved as a precursor in the synthesis of the antibiotic in question.

We wish to thank Drs A. L. Demain and S. Yankofsky for critical reading of the manuscript, V. Kuper for technical assistance and S. Linder for typing. The research was supported by a grant from the United States Israel Binational Science Foundation (BSF), Jerusalem, Israel.

\section{REFERENCES}

Aharonowitz, Y. \& Demain, A. L. (1977). Influence of inorganic phosphate and organic buffers on cephalosporin production by Streptomyces clavuligerus. Archives of Microbiology 115, 169-173.

Demain, A. L. (1957). Inhibition of penicillin formation by lysine. Archives of Biochemistry and Biophysics 67, 244-245.

Drew, S. W. \& Demain, A. L. (1977). Effect of primary metabolism on secondary metabolism. Annual Review of Microbiology 31, 343-356.

Gross, W. \& Burkhardt, K. L. (1973). Multiple transport systems for basic amino acid transport in Streptomyces hydrogenans. Biochimica et biophysica acta 298, 437-445.

Higgens, C. E. \& Kastner, R. E. (1971). Streptomyces clavuligerus sp. nov., a $\beta$-lactam antibiotic producer. International Journal of Systematic Bacteriology 21, 326-331.

Kern, B. A., Hendlin, D. \& Inamine, E. (1980). Llysine $\varepsilon$-aminotransferase involved in cephamycin C synthesis in Streptomyces lactamdurans. Antimicrobial Agents and Chemotherapy 17, 679-685.

KirkPatrick, J. R., DoOlin, L. E. \& Godfrey, O. W. (1973). Lysine biosynthesis in Streptomyces lipmanii: implication in antibiotic biosynthesis. Antimicrobial Agents and Chemotherapy 4, 542-550.

KohsaKa, M. \& Demain, A. L. (1976). Conversion of penicillin $\mathrm{N}$ to cephalosporin(s) by cell free extracts of Cephalosporium acremonium. Biochemical and Biophysical Research Communications 70, 465-473.

Luengo, J. M., Revilla, G., Lopez, M. J., VillaNUEVA, J. R. \& MARTIN, J. F. (1980). Inhibition and repression of homocitrate synthase by lysine in Penicillium chrysogenum. Journal of Bacteriology 144, 869-876.

Masurekar, P. S. \& Demain, A. L. (1972). Lysine control of penicillin biosynthesis. Canadian Journal of Microbiology 18, 1045-1048.

Masurekar, P. S. \& Demain, A. L. (1974). Impaired penicillin production in lysine regulatory mutants of Penicillium chrysogenum. Antimicrobial Agents and Chemotherapy 6, 366-368.

Mendelovitz, S. \& Aharonowitz, Y. (1982). Regulation of cephamycin C synthesis, aspartokinase, dihydropicolinic acid synthetase, and homoserine dehydrogenase by aspartic acid family amino acids in Streptomyces clavuligerus. Antimicrobial Agents and Chemotherapy 21, 74-84. 
SANo, K. \& ShIIo, I. (1970). Microbial production of L-lysine. III. Production by mutants resistant to $S$-(2-aminoethyl)-L-cysteine. Journal of General and Applied Microbiology 16, 373-391.

ShabTai, Y., Rosenberg, E. \& Kindler, S. H. (1975). Aspartokinase isoenzymes of the fruiting Myxobacterium Myxococcus xanthus. Biochimica et biophysica acta 403, 345-354.

Shiro, I. \& Mryajima, R. (1969). Concerted inhibition and its reversal by end product of aspartate kinase in
Brevibacterium flavum. Journal of Biochemistry $\mathbf{6 5}$, 849-859.

Stadtman, E. R., Cohen, G. N., Le-Bras, G. \& DE RoBichon-SzulmajsTER, H. (1961). Feedback inhibition and repression of aspartokinase activity in Escherichia coli and Saccharomyces cerevisiae. Journal of Biological Chemistry 236, 2033-2038.

Umbarger, H. E. (1978). Amino acid biosynthesis and its regulation. Annual Review of Biochemistry 47, 533606. 